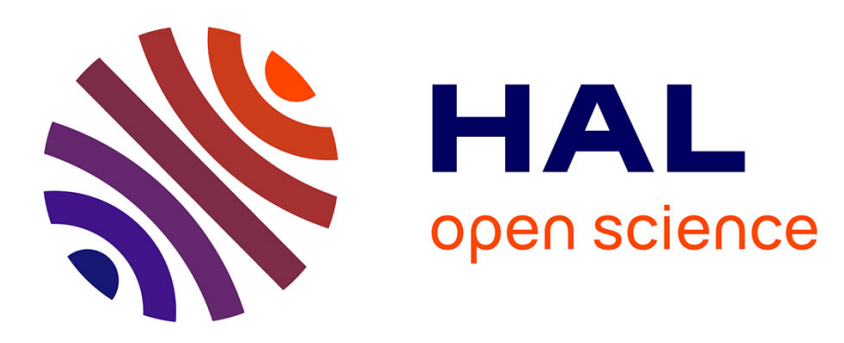

\title{
Comprehensive modelling study of singlet exciton diffusion in donor-acceptor dyads: when small changes in chemical structure matter
}

Giacomo Londi, Rishat Dilmurat, Gabriele D'avino, Vincent Lemaur, Yoann Olivier, David Beljonne

\section{To cite this version:}

Giacomo Londi, Rishat Dilmurat, Gabriele D'avino, Vincent Lemaur, Yoann Olivier, et al.. Comprehensive modelling study of singlet exciton diffusion in donor-acceptor dyads: when small changes in chemical structure matter. Physical Chemistry Chemical Physics, 2019, 21 (45), pp.25023-25034. 10.1039/c9cp05201a . hal-03011506

\author{
HAL Id: hal-03011506 \\ https://hal.science/hal-03011506
}

Submitted on 3 Dec 2020

HAL is a multi-disciplinary open access archive for the deposit and dissemination of scientific research documents, whether they are published or not. The documents may come from teaching and research institutions in France or abroad, or from public or private research centers.
L'archive ouverte pluridisciplinaire HAL, est destinée au dépôt et à la diffusion de documents scientifiques de niveau recherche, publiés ou non, émanant des établissements d'enseignement et de recherche français ou étrangers, des laboratoires publics ou privés. 


\title{
Comprehensive Modelling Study of Singlet Exciton Diffusion in Donor-Acceptor Dyads: When Small Changes in Chemical Structure Matter
}

Giacomo Londi ${ }^{a}$, Rishat Dilmurat ${ }^{a}$, Gabriele D'Avino $^{b}$, Yoann Olivier $^{a}$ and David Beljonne ${ }^{a}$

a) Laboratory for Chemistry of Novel Materials, University of Mons, Place du Parc, 20, 7000 Mons, Belgium

b) Institut Néel, CNRS and Grenoble Alpes University, 38042, Grenoble, France

\begin{abstract}
We compare two small $\pi$-conjugated Donor-Bridge-Acceptor organic molecules differing only by the number of thiophene rings in their bridging motifs (1 ring in 1; 2 rings in 2) and for which significantly different photovoltaic efficiencies have been reported in fullerene-based bulk heterojunction organic solar cells. The focus here is primarily on the use of fully atomistic modelling approaches to assess the origin for the reported differences in singlet exciton diffusion, held responsible for the improved photovoltaic response of 1 compared to 2. By combining Force Field Molecular Dynamics and Micro Electrostatic schemes to Time Dependent Density Functional Theory and Kinetic Monte Carlo simulations, we dissect the nature of the lowest electronic excitations in amorphous thin films of these molecules and model the transport of singlet excitons across their broadly disordered energy landscapes. In addition to a longer excited-state lifetime associated with a more pronounced intramolecular charge-transfer character, our calculations reveal that singlet excitons in $\mathbf{1}$ are able to funnel through long-distance hopping pathways, presumably as a result of the less anisotropic shape of the molecule favouring long-range 3D transport.
\end{abstract}




\section{Introduction}

Bulk heterojunction (BHJ) organic solar cells (OSCs) based on small molecules appear as compelling alternatives to polymer-based OSCs thanks to their peculiar benefits, including high purity, low molecular weight, well-defined and tunable structure, ease of synthesis and processability(1). Recently, the highest concerted power conversion efficiency (PCE) of 15.0\% was achieved for a tandem structure that combines both fullerene- and non-fullerene based OSCs, setting the new world record(2) and surpassing the previous PCE value of 14.2\%(3). These tremendous breakthroughs stem from engineering and innovation processes towards reducing or minimizing the mechanism losses that affect dramatically the performances of an OSC. Small modifications in the primary chemical structure of the electro-active molecules can turn into large changes in their photo-physical behavior and the resulting optoelectronic response at the device dimensions. We report such an example here, where the focus is on the nature and dynamics of singlet excitations in two small $\pi$-conjugated organic molecules, referred to as $\mathbf{1}$ and $\mathbf{2}$ in Figure 1.
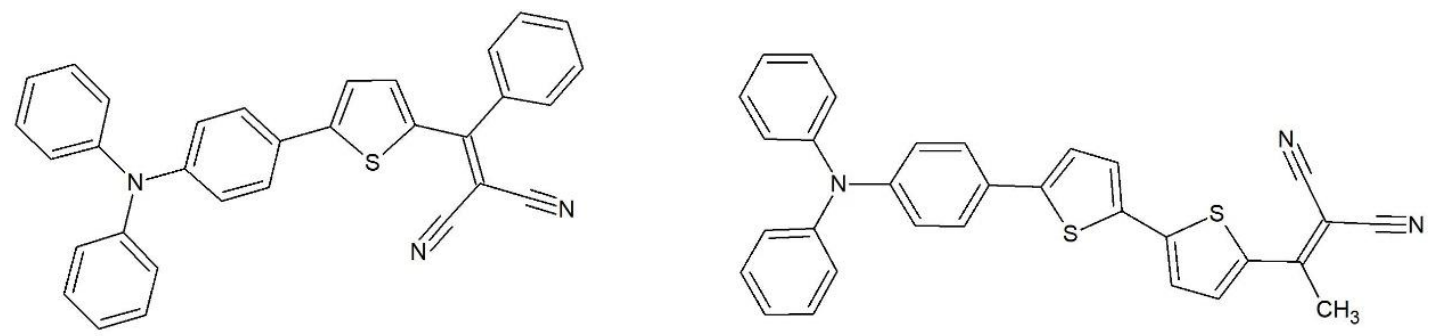

Figure 1: Chemical structures of 1 (left) and 2 (right).

These $\pi$-conjugated molecules belong to the class of push-pull systems, which in the last decades have found applications as active layers in organic devices, such as dye-sensitizing solar cells (DSSC)(4), fieldeffect transistors (OFET)(5), light-emitting diodes (OLED)(6) and photovoltaic (OPV)(7) cells. Such molecules were designed with the purpose of introducing a partial intramolecular charge transfer (ICT) character into the lowest exciton states. As these likely feature reduced Coulomb binding energy associated with larger electron-hole separation, they should also be more prompt to dissociation into separated charges at the interface with fullerene electron acceptors. The two D- $\pi$-A molecules both comprise a triphenylamine (TPA) fragment, acting as an electron donor (D), and a dicyanovinylene moiety (DCV), acting as an electron acceptor (A), linked through either a thiophene (in 1) or a bithiophene (in 2) $\pi$-linker. Electronic excited states in these molecules can be pictured as resulting from the mixing between Frenkel excitations (FE) on the $\pi$-conjugated spacer and through-bridge intramolecular D-A charge-transfer (CT) ones. While admixture of CT configurations would red-shift the excitation energies, overlapping electron-hole configurations predominantly feed the oscillator strength, hence enabling large absorption cross-sections in the visible and solar light harvesting. The synthesis of the two dyes, their electrochemical and time-resolved 
photoluminescence (PL) characterization along with OSC device fabrication and testing have been reported elsewhere(8-10).

Because it features a more extended conjugated backbone, one would anticipate that $\mathbf{2}$ has a significantly red-shifted optical absorption yielding better overlap with the solar emission spectrum and should thus outperform 1 when used as a donor in a BHJ solar cells. It turns out, however, that this is not the case, with significantly larger PCE values reported for $1(>5 \%)$ compared to $\mathbf{2}(\sim 2 \%)$ in vacuum-evaporated single solar cells using fullerenes as acceptors. Changes in morphology can be ruled out, as the same trend is observed irrespective of whether the active layer is solution-processed or vacuum-processed. Instead, it was suggested in Ref.(10) that this improvement at least partly arises from a more efficient singlet exciton diffusion in 1. As a matter of fact, the reported exciton diffusion length, that is the distance covered by the singlet excitons during their lifetime, was measured using PL quenching experiments to be significantly larger in $\mathbf{1}(\sim 26 \mathrm{~nm})$ compared to $\mathbf{2}(\sim 10 \mathrm{~nm})$. This result was attributed to the concerted effects of the longer exciton lifetime, $\sim 1.6 \mathrm{~ns}$ in $\mathbf{1}$ versus less than $1 \mathrm{~ns}$ in $\mathbf{2}$, together with the reduced energetic disorder in the smaller molecule. We note that typical values for singlet exciton diffusion lengths in organic semiconductors span the range 5-20 $\mathrm{nm}$ and are often limited by static conformational and/or electrostatic disorder(11,12).

Here, to gain an in-depth microscopic picture for the relationships between primary chemical structure and excited-state properties, we performed a fully atomistic modelling study of the energetics and dynamics of singlet electronic excitations in solid films of $\mathbf{1}$ and $\mathbf{2}$. More specifically, we employed classical Molecular Dynamics (MD) to simulate large scale morphologies of the two donors, in order to sample the conformational space in the amorphous (and crystalline) state. MD morphologies were then employed in time dependent density functional theory (TD-DFT) and classical microelectrostatic (ME) calculations that allowed the assessment of the nature of the relevant electronic excitations and their energy landscape, fully accounting for environmental effects in the solid phase. The results of this combined MD/TD-DFT/ME approach, together with excitonic intermolecular couplings, were then used in kinetic Monte Carlo (KMC) simulations of singlet exciton diffusion, based on the semi-classical Marcus-Levich-Jortner (MLJ) energy transfer rates. Our results confirm the experimental finding that $\mathbf{1}$ has both larger exciton diffusion constant and diffusion length with respect to 2 , a feature that arises from the combined effect of extended lifetime and the higher likelihood of long-range percolation pathways in the less anisotropic molecule 1, promoting 3D transport. 


\section{Theoretical methodology}

\subsection{Force-Field Molecular Dynamics Simulations}

In order to model the morphology of these materials, classical force field (FF) MD simulations were carried out. The employed FF was derived from Dreiding(13), while re-parameterizing the soft inter-ring torsional potentials (see ESI Figure S1 and S2) against reference MP2/cc-pVDZ calculations, as required for an accurate description of the conformational degrees of freedom. Amorphous phases of the two compounds (from now on 1a and 2a) were built using the amorphous builder tool of Materials Studio (MS) 6.0 package, which implements periodic boundary conditions. The simulations protocol consisted of the following steps:

1) Creation of an initial configuration of 1000 molecules in a large 3D periodic cubic box (initial density $\sim 0.2 \mathrm{~g} \mathrm{~cm}^{-3}$ ); 2) a MD run in the canonical (NVT) ensemble at $1000 \mathrm{~K}$ for $200 \mathrm{ps}$ to randomize molecular orientations; 3) a MD run in the isothermal-isobaric (NPT) ensemble at $1000 \mathrm{~K}$ for $1 \mathrm{~ns}$, compressing the sample by progressively increasing the pressure from $1 \mathrm{~atm}$ to $1 \mathrm{GPa}$; 4 ) a NPT-MD run at $1 \mathrm{GPa}$ for 600 ps, gradually cooling down the system from 1000 to $298 \mathrm{~K}$; 5) a NPT-MD run at $298 \mathrm{~K}$ for $800 \mathrm{ps,} \mathrm{releasing}$ the pressure from $1 \mathrm{GPa}$ to $1 \mathrm{~atm}$; and finally 6) a NPT-MD run for $2.5 \mathrm{~ns}$ at $298 \mathrm{~K}$ and $1 \mathrm{~atm}$. In addition, for $\mathbf{1}$, a crystalline $8 \times 6 \times 5$ triclinic supercell (from now on $\mathbf{1 x}$ ) was generated based on the experimentally resolved crystallographic unit cell(14), in order to reach a comparable size in term of numbers of molecules within the simulation box (960 molecules for $1 \mathbf{x}$ and 1000 molecules for 1a). The crystalline supercell was submitted to the following steps: 1) a NVT-MD run for 200 ps run at $298 \mathrm{~K}$; 2) a NPT-MD run for $2.5 \mathrm{~ns}$ at $298 \mathrm{~K}$ and $1 \mathrm{~atm}$. For both the amorphous and crystalline NPT-MD final runs of $2.5 \mathrm{~ns}, 500$ ps were needed for equilibration and $2 \mathrm{~ns}$ for production, saving snapshots every 5 ps along the trajectory. The Nosé-Hoover thermostat and the Berendsen barostat (for NPT runs) were adopted. The electrostatic interactions were taken into account by calculating the electrostatic potential (ESP) derived atomic charges on the isolated molecule at the MP2 level. MP2 calculations were performed with the GAUSSIAN09(15) suite, while MS 6.0 was adopted for the FF MD simulations.

The reparametrized FF was first benchmarked against experimental data by running simulations on 1x. Our NPT-MD simulations at standard conditions yield a density $d_{M D}=1.23 \mathrm{~g} \mathrm{~cm}^{-3}$, in perfect agreement with the experimental value of $d_{E X P}=1.24 \mathrm{~g} \mathrm{~cm}^{-3}$. Also, the predicted cell parameters compare very favorably with the measured crystallographic data, with relative error of less than $2 \%$ (see Table T1 in ESI). The densities of amorphous samples were $d_{M D}=1.02 \mathrm{~g} \mathrm{~cm}^{-3}$ for $\mathbf{1 a}$ (lower as expected, if compared to crystalline density) and $d_{M D}=0.97 \mathrm{~g} \mathrm{~cm}^{-3}$ for $\mathbf{2 a}$. Moreover, the orientational order in the amorphous samples was assessed by computing the second-rank order parameter $P_{2}=\left\langle\frac{3 \cos ^{2} \theta-1}{2}\right\rangle$, where $\theta$ is the angle 
between the long molecular axis and a given reference axis and angular brackets denote the average over the production MD trajectory. We obtained $P_{2}=-0.001$ for $1 \mathbf{a}$ and $P_{2}=0.009$ for 2a, testifying the isotropic nature of the amorphous samples.

\subsection{Excited-State Energetics}

Electronic structure calculations were performed to track the excited-state energetics for molecular snapshots extracted along the MD trajectories. In the DFT framework, global-hybrid functional, such as the popular B3LYP(16) and PBE0(17), are well-known to present shortcomings for the description of CT states in $\pi$-conjugated molecules. In addition to the self-interaction error (SIE) and the delocalization one $(18,19)$, they suffer a lack of the correct asymptotic behavior in the exchange-correlation energy functional. The latter is recovered in range-separated hybrid (RSH) functionals(20) adopted in the present study. Specifically, the optimally-tuned (OT) RSH functional $\omega \mathrm{B} 97 \mathrm{X}-\mathrm{D}(21,22)$ was employed. Moreover, according to the "gap tuning" procedure $(23,24)$, the $\omega$ value was finely tuned in order to match, within 50 meV of tolerance, the HOMO energy with the molecular vertical ionization potential (IP) and the LUMO energy with the vertical electron affinity (EA) of the molecule. Optimized $\omega$ values in RSH functionals are usually strongly system-dependent, in particular for $\pi$-conjugated molecules, yet turn out to have very similar values here for $\mathbf{1}\left(\omega=0.139 \mathrm{Bohr}^{-1}\right)$ and $\mathbf{2}\left(\omega=0.141 \mathrm{Bohr}^{-1}\right)$. These values were kept fixed throughout the DFT and TD-DFT simulations, carried out using GAUSSIAN09(15) with the 6-31G(d,p) basis set, except where otherwise specified.

The effect of the polarizable molecular environment on intra-molecular excitations energies was accounted for by means of classical microelectrostatic (ME) calculations $(25,26)$, following our previous work(27). In a nutshell, ME allows the calculation of a perturbative correction ( $\Delta$, the exciton polarization energy) to gas-phase TD-DFT lowest energy excitation $\left(\mathrm{S}_{1}\right)$ that can be decomposed into an electrostatic and an induction contribution:

$\Delta=\Delta_{E}+\Delta_{I}$

where $\Delta_{E}$, the electrostatic component, accounts for the interaction of the exciton and the charge densities of the surrounding ground-state molecules, and the induction term $\Delta_{I}$ describes the interaction between the excitation and the microscopic dipoles induced in the medium. Self-consistent ME calculations were performed for spherical clusters of $4 \mathrm{~nm}$ radius centred around the molecule of interest. The ME model was parametrized with gas-phase ESP(28) atomic charges calculated at the DFT and TD-DFT $\omega$ B97X-D/6$311++\mathrm{G}(\mathrm{d}, \mathrm{p})$ level for the ground $\left(\mathrm{S}_{0}\right)$ and the excited $\left(\mathrm{S}_{1}\right)$ state, respectively. The polarizability tensor of 
the ground-state molecule, computed at the same level of theory mentioned above, was adopted to describe both $\mathrm{S}_{0}$ and $\mathrm{S}_{1}$.

TD-DFT calculations were repeated for all individual molecules extracted from the last MD frame and also for 20 molecules (extracted from 400 snapshots) followed along the 2 ns trajectory, thus allowing to build some statistics in space and time. The fluctuations of $\mathrm{S}_{1}$ excitation energy can be quantified by the standard deviation $\sigma_{\text {total }}$ of its distribution. These fluctuations can be split into two different contributions, according to the relevant timescales of the fluctuations: a static contribution (i.e. presenting negligible variations over the time scales characterizing exciton lifetimes, $\sim 1 \mathrm{~ns}$ ), which results from the different environment experienced by each molecule, and a dynamic contribution, associated with faster molecular motions:

$\sigma_{\text {total }}=\sqrt{\sigma_{\text {static }}^{2}+\sigma_{\text {dynamic }}^{2}}$

$\sigma_{\text {total }}$ is obtained as the full $\mathrm{S}_{1}$ standard deviation for all molecules extracted from the last MD frame. In order to assess the dynamic contribution, 20 molecules were randomly selected and their excitation energies tracked along the $2 \mathrm{~ns}$ MD trajectory. The fluctuations around their individual mean lead to the dynamical variance:

$\sigma_{\text {dynamic }}^{2}=\frac{1}{n} \sum_{i, k}\left(E_{i k}-\overline{E_{k}}\right)^{2}$

where $E_{i k}$ is the $\mathrm{S}_{1}$ energy of molecule $k$ at time $i, \overline{E_{k}}$ is the mean energy of molecule $k$, and $n$ is the number of samples. $\sigma_{\text {static }}$ is then obtained by subtraction from Equation (2).

\subsection{Singlet Exciton Hopping Rates and Diffusion}

Singlet exciton diffusion in the amorphous phase of two materials was reproduced using a hopping transport model, which is fully justified owing to the much smaller excitonic couplings $(<100 \mathrm{meV})$ compared to the total relaxation energies $(\sim 500 \mathrm{meV})$. The hopping rate was computed using the non-adiabatic semiclassical MLJ expression that accounts for quantum tunneling effects(29):

$\kappa_{i f}=\frac{2 \pi}{\hbar} V_{i f}^{2} \sqrt{\frac{1}{4 \pi \lambda_{s} k_{B} T}} \times \sum_{n}\left\{\exp \left(-S_{\text {eff }}\right) \frac{S_{\text {eff }}^{n}}{n !} \times \exp \left[-\frac{\left(\Delta G_{i f}^{0}+\lambda_{s}+n \hbar \omega_{e f f}\right)^{2}}{4 \lambda_{s} k_{B} T}\right]\right\}$

where $\kappa_{i f}$ is the exciton hopping rate between molecular sites $i$ and $f ; V_{i f}$ is the corresponding excitonic coupling; $\lambda_{s}$ is the external reorganization energy; $k_{B}$ is the Boltzmann constant; $T$ is the temperature; $\Delta G_{i f}^{0}$ is the free Gibbs energy difference involved in the hopping mechanism; $\hbar \omega_{\text {eff }}$ is the energy of an effective high-frequency intramolecular vibrational mode (the overarching carbon-carbon stretching) that assists the 
exciton hopping; $S=\lambda_{i} /\left(\hbar \omega_{e f f}\right)$ is the Huang-Rhys factor, which measures the internal reorganization energy $\lambda_{i}$.

Our atomistic calculations were used to provide reliable parameters for Equation (4). Site energies were drawn from a Gaussian distribution with standard deviation $\sigma_{\text {static }}$ and then used to compute inter-site free energy differences $\Delta G_{i f}^{0}$. The dynamic disorder $\sigma_{\text {dynamic }}$ is instead related to the total reorganization energy, and specifically for linear exciton-phonon coupling to a bath of classical harmonic modes:

$\lambda_{\text {total }}=\frac{\sigma_{d y n a m i c}^{2}}{k_{B} T}$

In turn, $\lambda_{\text {total }}$ comprises two different contributions:

$\lambda_{\text {total }}=\lambda_{i}+\lambda_{s}$

where the internal part, $\lambda_{i}$, arises from the difference in the equilibrium geometries between the molecule ground state $\left(\mathrm{S}_{0}\right)$ and the first excited state $\left(\mathrm{S}_{1}\right)$, while the external one, $\lambda_{s}$, is due to the structural relaxation of the surrounding medium upon energy transfer. Since high-frequency modes are quantum in nature, $\lambda_{i}$ can be more safely estimated with the 4-point approach based on DFT/TD-DFT calculations in the gasphase, namely:

$\lambda_{i}=E\left(S_{1} \mid r_{0}\right)-E\left(S_{1} \mid r_{1}\right)+E\left(S_{0} \mid r_{1}\right)-E\left(S_{0} \mid r_{0}\right)$

where $E\left(S_{m} \mid r_{n}\right)$ indicated the energy of the state $S_{m}$ at the geometry optimized for the state $S_{n}$. Please note that the geometry optimization in the excited state was performed starting from the ground state geometry and freezing the soft torsional angles, in order to account only for the contribution from the high-frequency modes (and thus avoid double-counting, since the low-frequency modes enter $\lambda_{i}$ through the classical MD simulations). The external contribution was then calculated by subtraction from the total reorganization energy, according to equations (5) and (6).

The excitonic couplings, $V_{i f}$, between molecules in the amorphous samples were computed at the TD-DFT level, resorting to the fragment excitation difference (FED) scheme(30). The molecular pairs (dimers) were selected according to a geometrical criterion, namely electronic couplings were computed for dimers with less than $20 \AA$ distance between their center of mass. The FED scheme is a diabatization procedure that relies on the calculation of the attachment density (the density of the electron promoted in the transition) and the detachment density (the hole density created in the transition)(31). The electronic couplings are obtained through a unitary transformation that yields the localized diabatic states by maximizing the excitation density difference between the two molecules involved in the energy transfer process. While 
exchange and overlap might contribute at short intermolecular distances, the Coulomb term is expected to dominate at long range, where it is expected to decay following the usual Förster-like $R^{-3}$ dipole-dipole interaction.

Finally, kinetic Monte Carlo (KMC) simulations employing the first reaction method (FRM) algorithm(32) were performed to assess exciton diffusion coefficient and length. Such a stochastic approach involves the following steps: (1) initialization of the system at time $t=0$; (2) calculation of the hopping rates from MLJ equation; (3) quantifying the time for hopping events between an initial $(i)$ and a final site $(f)$ using the following equation:

$\Delta t_{i f}=-\frac{\ln (r)}{\kappa_{i f}}$

where $r$ is a random number generated between 0 and 1 and $\kappa_{i f}$ is MLJ hopping rate; (4) selecting the event with the smallest $\Delta t_{i f}$ for the exciton to hop and update its position; (5) updating the time $t=t+\Delta t_{i f}$ if $t$ is less than the simulation time (10 ns), else stop the simulation. In order to obtain statistically relevant results, and because of the sizeable energetic disorder, a large number of simulations were required (i.e. one million). From the time-dependent mean-square displacements, averaged singlet exciton diffusion coefficient and diffusion length, $L_{d}$, are related through:

$L_{d}=\sqrt{D \tau_{1}}$

with $\tau_{1}$ the exciton lifetime.

\section{Results and Discussion}

\subsection{Excited-state Electronic Structure}


To get insight into the nature of the lowest electronic excitations in the two molecules, we first performed gas-phase TD-DFT calculations at the ground-state geometries. Multiple metrics allow assessing the character of electronic excitations $(33,34)$. Here, we have opted for the overlap, $\Phi_{s}$, between the hole and electron densities shown in Figure 2 for the two molecules. Pure CT excitations correspond to nonoverlapping hole and electron density $\left(\Phi_{s}=0\right)$, while fully FE excitations instead lead to $\Phi_{s}=1$. The CT or FE character of the excitation depends strictly on the molecular geometry and its fluctuation around equilibrium, as well as on the choice of the exchange-correlation functional.
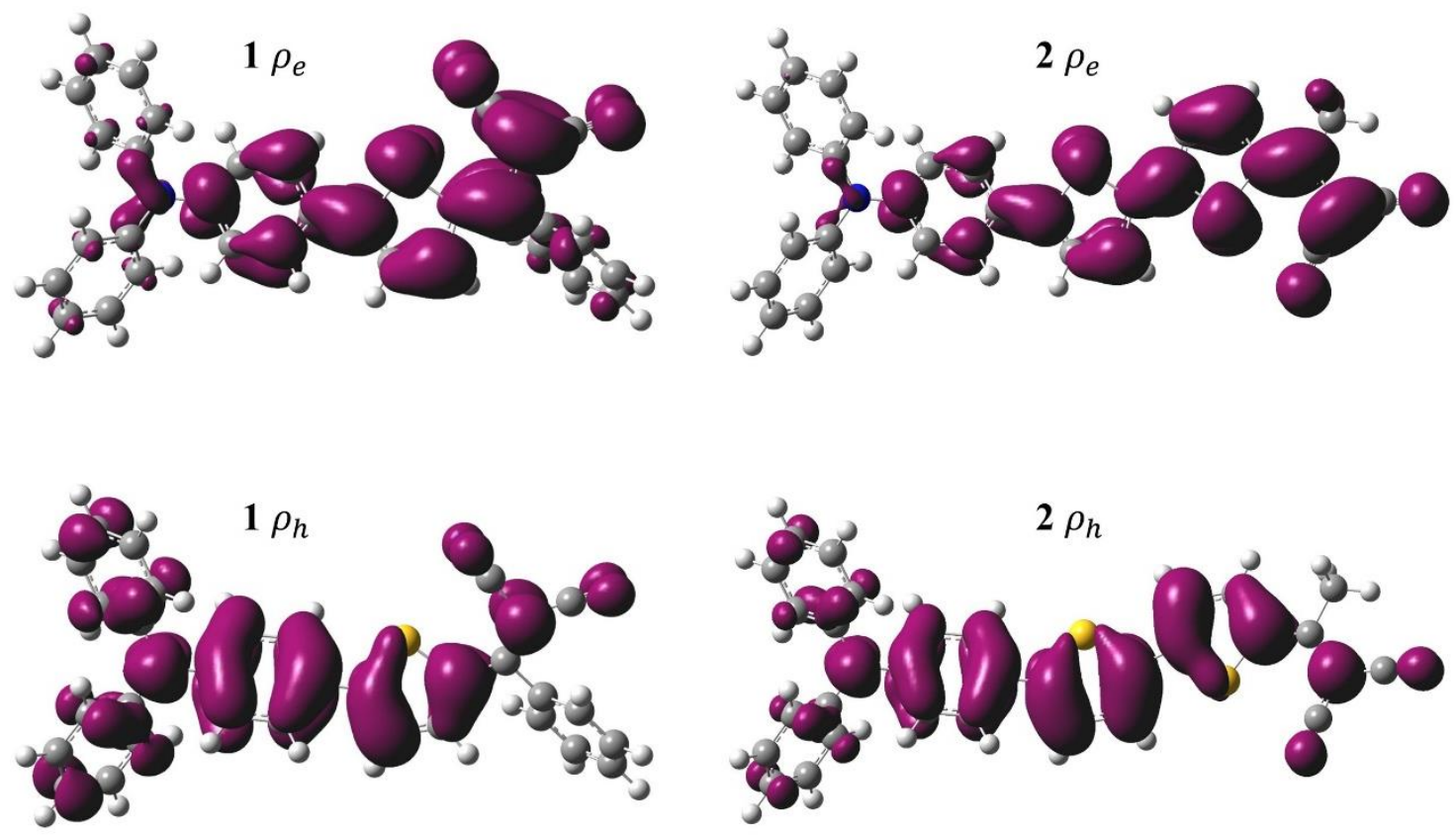

Figure 2: Hole $\left(\rho_{h}\right)$ and electron $\left(\rho_{e}\right)$ densities calculated in the attachment/detachment formalism for $\mathrm{S}_{1}$ in 1 (left) and 2 (right), as obtained at the OT-RSH TD-DFT level $(\omega$ B97X-D/6-31G(d,p)) based on gasphase equilibrium ground-state geometries.

The hole and electron density plots in Figure 2 show that $S_{1}$ has a mixed $\pi$ - $\pi^{*} / C T$ character(35), with both hole and electron density spreading over most of the $\pi$-system in both compounds. This translates into $\Phi_{S}$ values of $\sim 0.66$ in 1 and $\sim 0.72$ in $\mathbf{2}$. Thus, extending the bridge from one to two thiophene rings slightly reduces the CT character of the electronic excitations. We next repeated the TD-DFT calculations on all molecules individually extracted from the last MD frame of the three investigated samples (the amorphous 1a and 2a and the crystalline 1x). The results, reported in Figure 3, show a broad distribution of the holeelectron overlap factors around the values predicted at the equilibrium geometries, clearly associated with thermal fluctuations in the conformation of the molecules. In particular, rotations around the single bonds connecting the donor to the bridge and the bridge to the acceptor (see Figure S3 in ESI) modulate the coupling between the donor and the acceptor groups of the molecule and, therefore, the amount of CT-FE admixture. It is interesting to point out that $\Phi_{s}$ distributions in wavefunction overlap are asymmetric, 
showing a long tail at low $\Phi_{s}$ values. As these are also low-energy conformations, we anticipate that the exciton energy landscape should show some degree of inhomogeneity with possible formation of trap states.
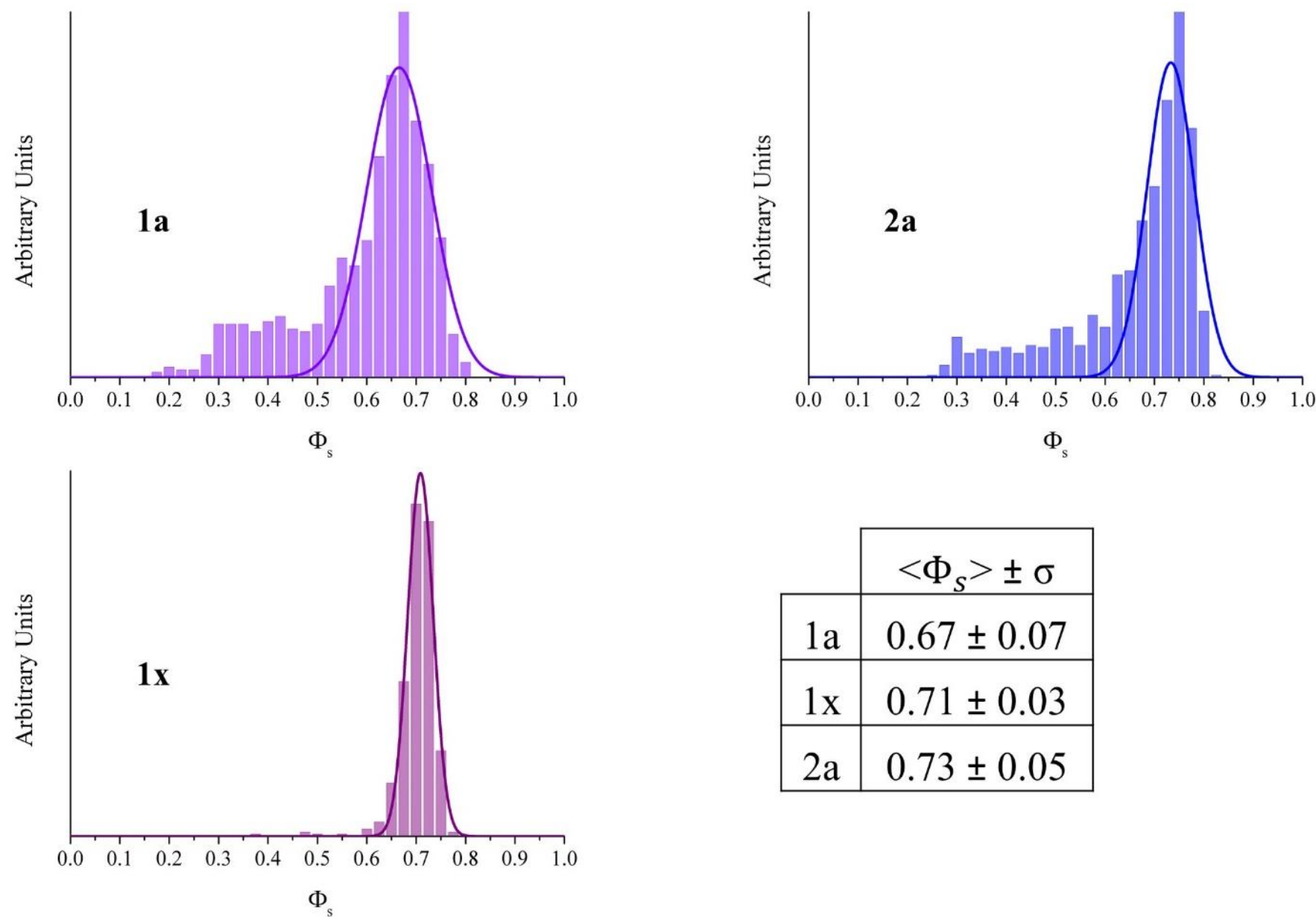

\begin{tabular}{|c|c|}
\cline { 2 - 2 } \multicolumn{1}{c|}{} & $\left\langle\Phi_{S}> \pm \sigma\right.$ \\
\hline $1 \mathrm{a}$ & $0.67 \pm 0.07$ \\
\hline $1 \mathrm{x}$ & $0.71 \pm 0.03$ \\
\hline $2 \mathrm{a}$ & $0.73 \pm 0.05$ \\
\hline
\end{tabular}

Figure 3: Room-temperature MD distributions of hole-electron density overlaps $\Phi_{s}$ in $\mathrm{S}_{1}$ for $\mathbf{1 a}, \mathbf{1 x}$ and $\mathbf{2 a}$. The averaged $\Phi_{s}$ values and their standard deviations are also reported in the table. Solid lines are Gaussian fits.

We further note that, as expected, the molecules in the long-range ordered $\mathbf{1 x}$ explore a much smaller region of the conformational space, hence the narrower $\Phi_{s}$ distribution. Very interestingly, Figure 4 convincingly demonstrates that the $\mathrm{S}_{1}$ excitation energy and its CT character are correlated, with larger CT admixture (lower $\Phi_{s}$ ) translating into red-shifted excitations. This is particularly pronounced for molecule 1, where conformations yielding the lowest excitation energies are predominantly CT-like, with $\Phi_{s}$ approaching 0.3. 


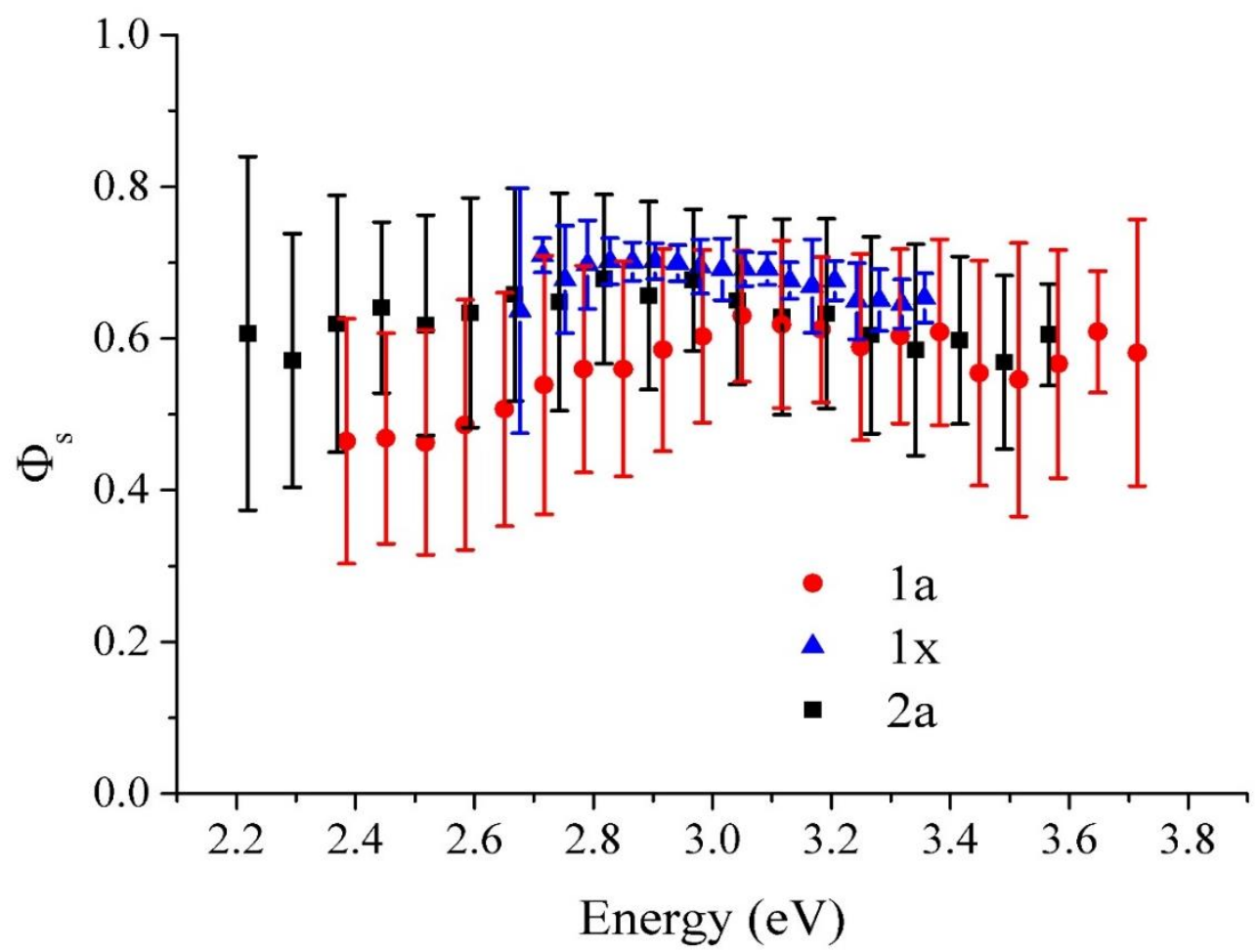

Figure 4: Hole-electron density overlap $\Phi_{s}$ versus transition energy to $S_{1}$, as obtained at the OT-RSH TD-DFT level on MD geometries for $\mathbf{1 a}$ (red dots), 1x (blue triangles) and $\mathbf{2 a}$ (black squares). Data set standard deviation at RT is represented by vertical bars.

From sampling of the configurational space at room-temperature, exciton Density of States (DOS) distributions were calculated for single molecules either in the gas phase or embedded in a fully atomistic electrostatic embedding. The results, portrayed in Figure 5, show broad, Gaussian-like, DOS primarily governed by changes in molecular conformation, with different standard deviations obtained in absence versus in presence of the polarizable environment $\left(\Delta \sigma_{\text {total }}<30 \mathrm{meV}\right)$. The electrostatic effects primarily contribute a shift of the distributions, by $0.17 \mathrm{eV}$ for $1 \mathbf{a}, 0.13 \mathrm{eV}$ for $1 \mathbf{x}$ and $0.10 \mathrm{eV}$ for $\mathbf{2 a}$. These small energy corrections reflect the reduced ionic character of the lowest electronic excitations - for the sake of comparison, excess positive or negative charges are typically stabilized by about $1 \mathrm{eV}$ energy corrections in polarizable molecular crystals(25,36,37). The most striking feature from Figure 5 is the rather similar mean values predicted in the amorphous phase of the two molecules, despite the more extended $\pi$-bridge in $\mathbf{2}$ compared to $\mathbf{1}$. This is ascribed to the increased CT character in the shorter molecule, as documented above, that compensates for its reduced conjugation length. 

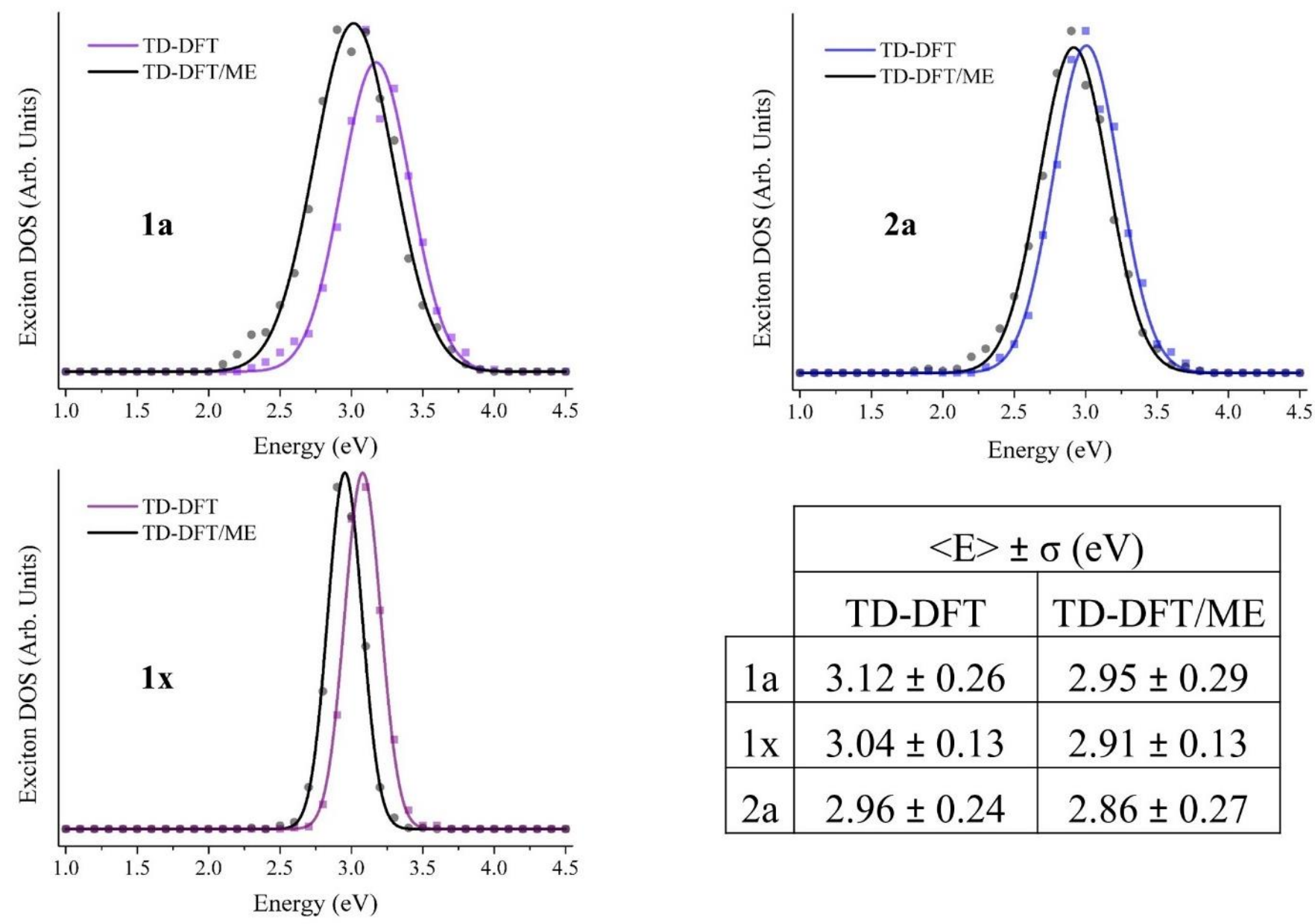

\begin{tabular}{|c|c|c|}
\cline { 2 - 3 } \multicolumn{1}{c|}{} & \multicolumn{2}{c|}{$<\mathrm{E}> \pm \sigma(\mathrm{eV})$} \\
\cline { 2 - 3 } \multicolumn{1}{c|}{} & TD-DFT & TD-DFT/ME \\
\hline $1 \mathrm{a}$ & $3.12 \pm 0.26$ & $2.95 \pm 0.29$ \\
\hline $1 \mathrm{x}$ & $3.04 \pm 0.13$ & $2.91 \pm 0.13$ \\
\hline $2 \mathrm{a}$ & $2.96 \pm 0.24$ & $2.86 \pm 0.27$ \\
\hline
\end{tabular}

Figure 5: Exciton Density of States (DOS) for the lowest electronic excitation based on gap-phase TD-DFT calculations (coloured) and accounting for electrostatic embedding TD-DFT/ME (in black). Averaged excitation energies and their standard deviations are also reported in the table. Solid lines are Gaussian fits.

We stress that these predictions both qualitatively and quantitatively match with thin-film optical absorption measurements reported in Ref.(9,10). There, the transition energies measured at $\lambda_{\max }$ for the two molecules almost coincide and the spectral lineshapes can be reasonably fitted with Gaussian functions of standard deviations close to those predicted by theory, although a quantitative comparison with experiment is hampered by the lack of quantum nuclear effects (i.e. Franck-Condon structure) and by the assumption of excitons strictly localized on molecular units in the calculations. Moreover, it should be stressed that these large $\sigma$ values refer to the total width, which encompasses static and dynamic disorder, see Table 1 below. As a side note, we remark that, besides its obvious narrower character, the center of the energy distribution predicted in the crystalline case is almost iso-energetic with that in the corresponding amorphous phase. While this might sound surprising at first glance, this is once again the result between the interplay of molecular conformation (on averaged more planar in the crystalline phase) and CT-FE mixing (reduced CT character in the crystal). We believe this is a potentially important result, as local crystalline domains 
embedded in an otherwise amorphous matrix usually act as trapping sites. This would not be the case for exciton transport in $\mathbf{1}$, which thus should be more resilient to positional disorder.

Another molecular characteristic that, in addition to being relevant for the excited-state lifetime and therefore the singlet exciton diffusion length, directly probes the nature of the electronic excited states is the radiative decay rate $\Gamma_{\text {rad }}$. This quantity may be related to the nature of the excited states in the two compounds, since a larger CT character would result in a smaller transition dipole moment and, hence, a smaller $\Gamma_{\text {rad }}$. From a perturbative treatment of light-matter interactions, the spontaneous emission rate $\Gamma_{\text {rad }}$ writes(38):

$\Gamma_{\text {rad }}=n \frac{\omega_{e g}^{3}\left|\vec{\mu}_{e g}^{2}\right|}{3 \pi \varepsilon_{0} \hbar c^{3}}$

where $\omega_{e g}$ is the transition frequency from the excited to the ground state, $\vec{\mu}_{e g}$ the transition dipole moment, $\varepsilon_{0}$ the dielectric vacuum permittivity, $\hbar$ the reduced Planck constant, $c$ the speed of light, and $n=\sqrt{\varepsilon_{r}}$ the refractive index of the medium, here set equal to 1.49 for a poly(methyl) acrylate (PMMA) matrix (for comparison to experiments reported in Ref.(10)). For the investigated three samples, the calculated radiative exciton lifetime, $\tau_{\text {rad }}$, averaged over all accessible conformations as explored by room-temperature MD simulations, amounts to $2.6 \mathrm{~ns}$ in $\mathbf{1 a}, 1.5 \mathrm{~ns}$ in $\mathbf{1 x}$ and $1.9 \mathrm{~ns}$ in $\mathbf{2 a}$. The radiative lifetimes are in line with expectations based on the character of the electronic exited states, with namely a larger value for 1 reflecting an increased CT contribution in the amorphous phase. They are also on par with the total lifetimes measured in amorphous films of the two dyes, respectively $2.6 \mathrm{~ns}$ and less than $1 \mathrm{~ns}$ in $\mathbf{1}$ and $\mathbf{2}$, respectively $(9,10)$. PL quantum yield measurements using an integrating sphere yield a value of $46 \%$ in a powder of 1(14) that, combined with the PL total lifetime, provides an experimental estimate for $\tau_{\text {rad }}$ of about $3.5 \mathrm{~ns}$, in fair agreement with the theoretical value of $2.6 \mathrm{~ns}$. This comforts us in the robustness of our theoretical methodology to properly reproduce the nature and energetics of singlet optical excitations in the molecular materials at hands. Actually, while we are here averaging over the full, broad, exciton DOS, it is likely that low-energy molecular sites contribute predominantly to the PL signal, after (partial) thermalization of the energy carriers.

\subsection{Singlet Exciton Hopping Rates}

Excited-state energetics enters the MLJ rates in three different ways: (i) the Gibbs free energy, simply equated to the static energetic disorder; (ii) the external, classical (low-frequency) and (iii) the internal, quantum (high-frequency) contributions to the nuclear reorganization energies. All these energies can be obtained from our atomistic simulations, provided some educated guess on time-scale separations. Namely, 
processes that are slow (fast) compared to hopping events were considered static (dynamic). From now on, we will analyze only the exciton diffusion properties for the amorphous pristine materials in order to provide a better comparison among our investigated samples. The calculated data that feed the MLJ equation for 1a and $\mathbf{2 a}$ are collected in Table 1.

Table 1: Calculated $\sigma_{\text {total }}, \sigma_{\text {static }}$ and $\sigma_{\text {dynamic }}$ values, along with $\lambda_{\text {total }}, \lambda_{i}$ and $\lambda_{s}$ ones. $\sigma_{E X P}$ values extracted from a Gaussian fit of the experimental absorption spectra are also indicated (from Ref. $(9,10)$ ).

\begin{tabular}{|c|c|c|c|c|c|c|c|}
\cline { 2 - 7 } \multicolumn{1}{c|}{} & $\boldsymbol{\sigma}_{\text {total }}(\mathrm{eV})$ & $\boldsymbol{\sigma}_{\text {EXP }}(\mathrm{eV})$ & $\boldsymbol{\sigma}_{\text {static }}(\mathrm{eV})$ & $\boldsymbol{\sigma}_{\text {dynamic }}(\mathrm{eV})$ & $\boldsymbol{\lambda}_{\text {total }}(\mathrm{eV})$ & $\boldsymbol{\lambda}_{\mathbf{i}}(\mathrm{eV})$ & $\boldsymbol{\lambda}_{\mathbf{s}}(\mathrm{eV})$ \\
\hline $\mathbf{1 a}$ & 0.294 & 0.25 & 0.270 & 0.115 & 0.514 & 0.245 & 0.269 \\
\hline $\mathbf{2 a}$ & 0.268 & 0.28 & 0.243 & 0.113 & 0.494 & 0.296 & 0.198 \\
\hline
\end{tabular}

The energetic disorder is primarily sourced by nuclear motions that are slow relative to exciton hopping, and thus static in nature. These likely involve large-amplitude changes in the conformation of the molecules, which are slowed down by packing effects in the solid. Much faster (internal) reorganizations in bond lengths mediated by high-frequency stretching/breathing modes and small (external) rearrangement around the intramolecular equilibrium geometry induced by the environment contribute with similar weights to the total reorganization energy.

We now discuss the excitonic couplings, $V_{i f}$, mediating singlet exciton hopping and the MLJ hopping rates, $\kappa_{i f}$. The FED values are plotted as a function of the intermolecular distance between centers of mass in Figure 6 top. For comparison, we show in Figure 6 bottom the corresponding couplings obtained by retaining only Coulomb interactions in the point dipole - point dipole approximation (PDA). As documented previously(30), this approximation fails at short distances where it produces erroneously large couplings. At long distances, FED and PDA show the expected inverse cubic distance dependence. 

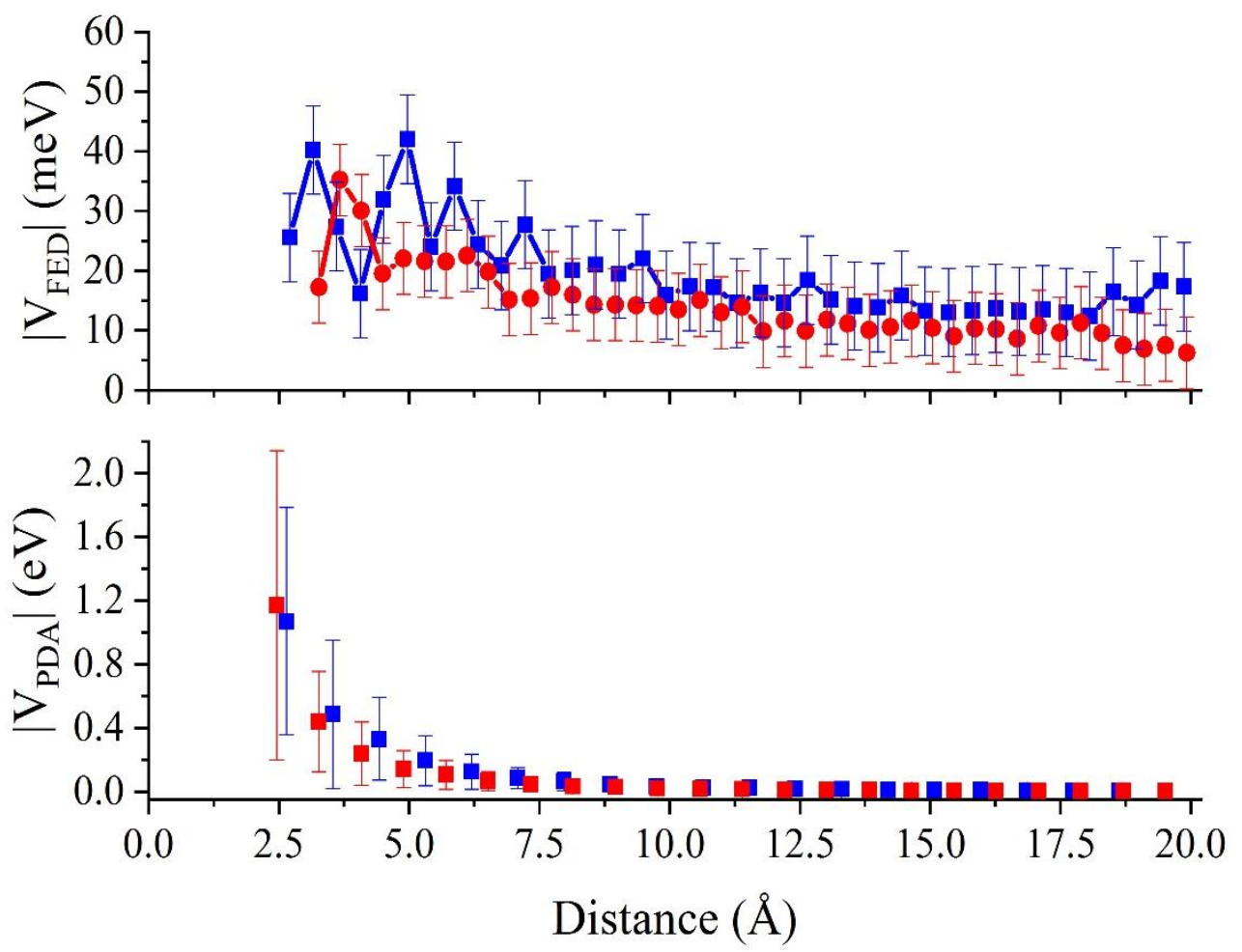

Figure 6: Excitonic coupling calculated with the FED scheme (top) and in the point dipole point dipole approximation (bottom) for the $\mathbf{1 a}$ (in red) and $\mathbf{2 a}$ (in blue). Data set standard deviation is represented by vertical bars.

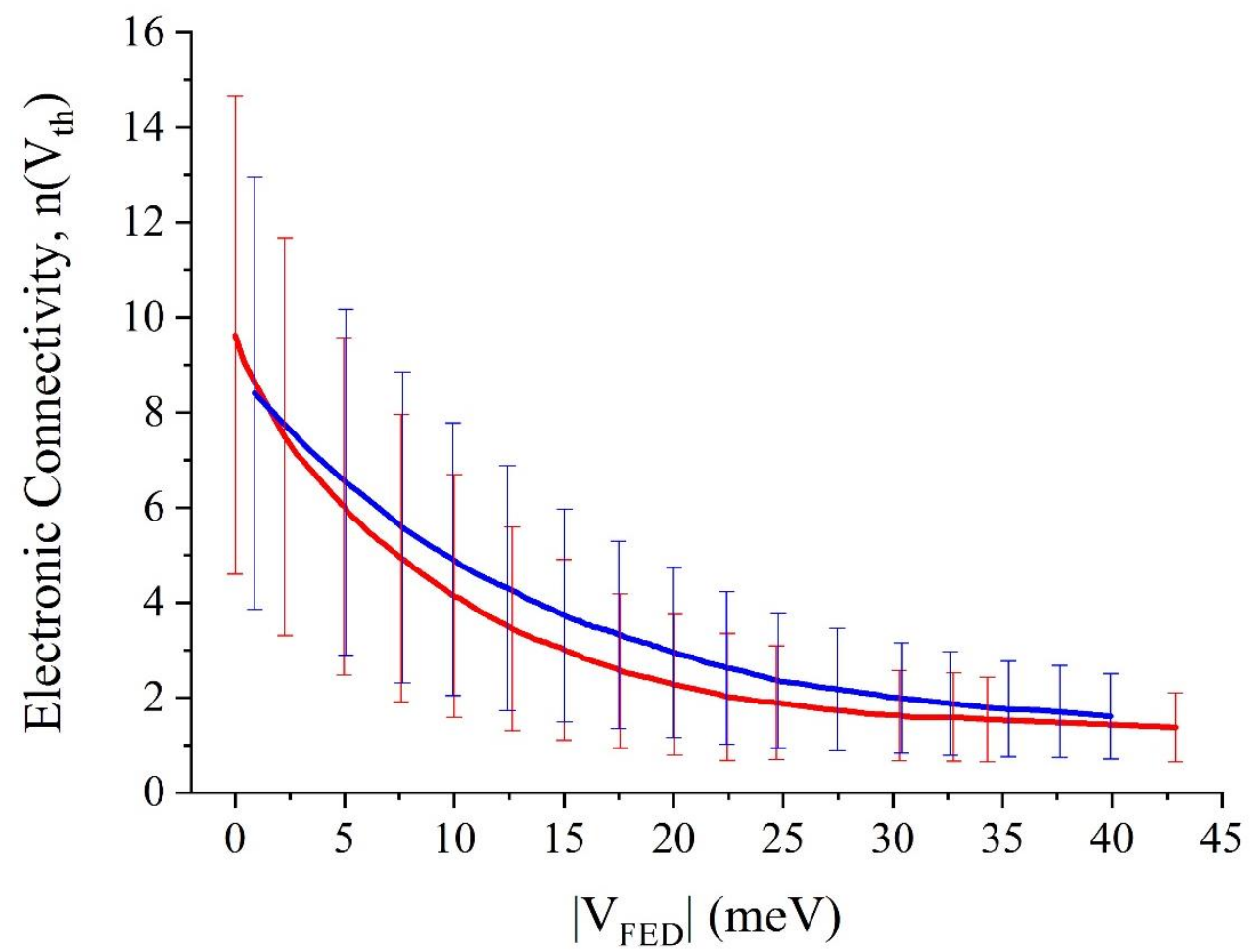

Figure 7: Electronic connectivity calculated with the FED scheme for $\mathbf{1 a}$ (in red) and 2a (in blue). Data set standard deviation is represented by vertical bars. 
A more useful metric for the interactions between molecules is the electronic connectivity or effective coordination number, defined as the number, $n\left(V_{t h}\right)$, of neighbor molecules $f$ coupled to a central molecule $i$ with a coupling $V_{i f}$ larger than a given threshold value $V_{t h}$. As expected, $n\left(V_{t h}\right)$ decreases with $V_{t h}$ to reach values close to zero for couplings larger than $\sim 40 \mathrm{meV}$, Figure 7 .

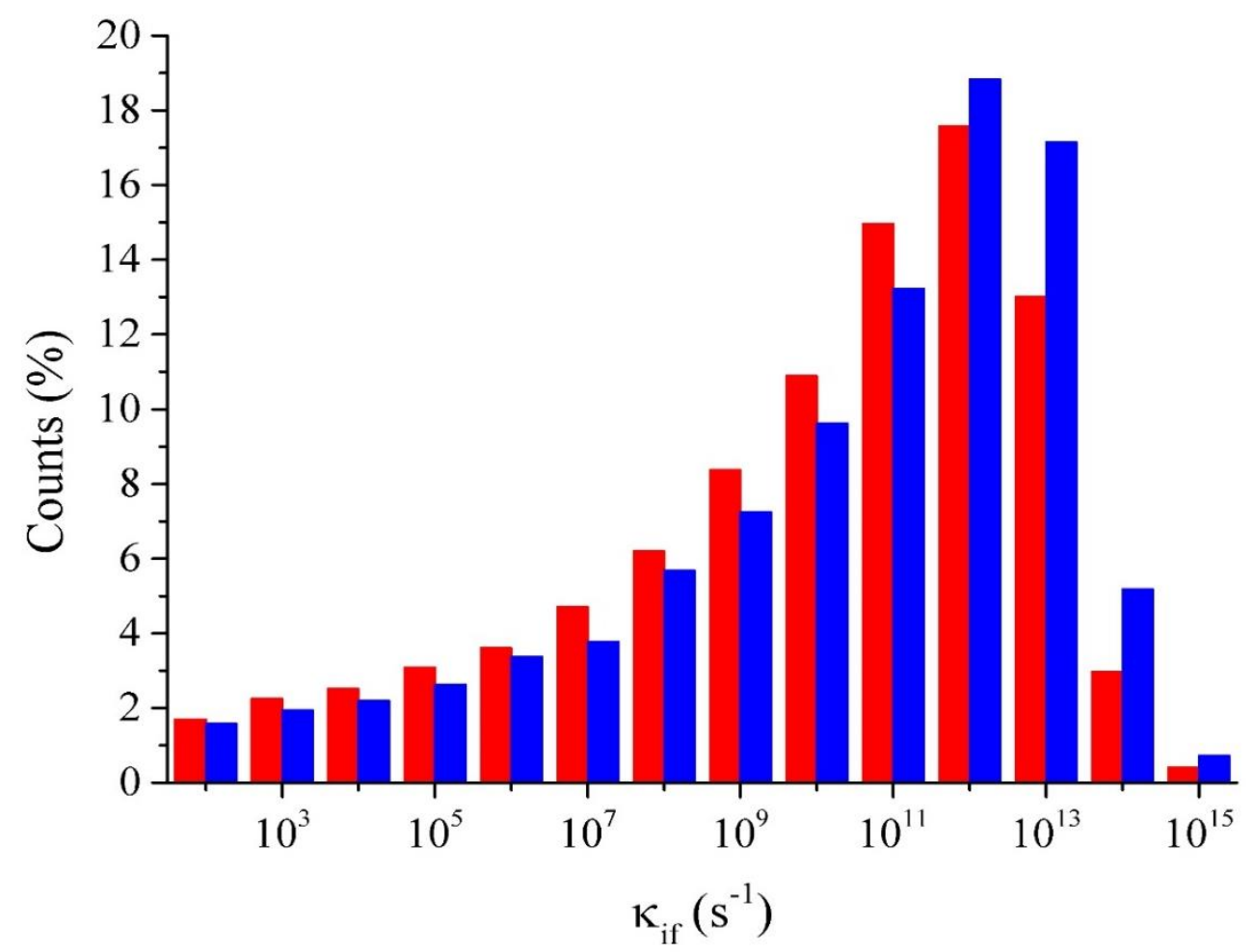

Figure 8: MLJ exciton hopping rates $\kappa_{i f}$ distribution calculated for $\mathbf{1 a}$ (in red) and $\mathbf{2 a}$ (in blue).

The two molecules show similar evolutions with, on average, 2-6 molecules displaying excitonic couplings in the range 10-25 meV. Combining these excitonic couplings with the internal and external reorganization energies listed in Table 1, we obtained the distributions of nearest neighbor exciton hopping rates, shown in Figure 8. These span about six orders of magnitude in timescales with a long tail at small $\kappa_{i f}$ corresponding to unlikely events associated with poorly connected molecules. For both molecules, the rate distributions peak at around $0.1-1 \mathrm{ps}^{-1}$, which is significantly faster than the exciton lifetimes, thus allowing for multiple jumps during the exciton lifespan.

Table 2: Calculated diffusion coefficient and the exciton diffusion length for $\mathbf{1 a}$ and $\mathbf{2 a .}$

\begin{tabular}{|c|c|c|}
\cline { 2 - 3 } \multicolumn{1}{c|}{} & Diffusion coefficient $\left(\mathrm{cm}^{2} \mathrm{~s}^{-1}\right)$ & Diffusion length $(\mathrm{nm})$ \\
\hline $\mathbf{1 a}$ & $1.53 \times 10^{-3}$ & 18 \\
\hline
\end{tabular}




\begin{tabular}{|l|l|c|}
\hline $2 \mathbf{a}$ & $1.28 \times 10^{-3}$ & 10 \\
\hline
\end{tabular}

\subsection{Singlet Exciton Diffusion}

KMC simulations were performed to model exciton dynamics. In a first set of simulations, we assumed as initial conditions a broad band excitation, that is all molecular sites were excited with the same probability irrespective of their energy. We start by analysing the time evolution of the average exciton energy. The results, reported in Figure 9, clearly show two different regimes. At early time after light absorption $(<10$ ps), there is a rapid and large (about half-an-eV) drop in energy attributed to non-equilibrium relaxation processes from initially hot energy carriers. At longer timescales, the system keeps cooling down at a much smaller pace, with no sign of full thermalization during the exciton lifetime. At this point, we can already conclude that exciton diffusion in these disordered molecular materials proceeds largely in a nonequilibrium manner. This is corroborated by the calculated mean square displacement over time ratio, which in the normal diffusion regime, equates the diffusion coefficient and should converge to a time-independent value in equilibrium conditions. Though this is an abuse of language, for the sake of simplicity, we will keep referring to diffusion coefficient in the following. With this caveat, it is interesting to notice that, for simulation times commensurate with the singlet lifecycle (1-2 ns), $D$ is slightly larger for $\mathbf{1}$ compared to $\mathbf{2}$, see Figure10a. We will return to this point later but the consequence of this difference in diffusion coefficient, together with the longer-lived singlet exciton, is molecule $\mathbf{1}$ showing a significantly larger exciton diffusion length than 2 , see Figure $10 \mathrm{~b}$ (where we used the measured total lifetimes). In view of the 
multifaceted aspect and complexity of the modelling approach, the calculated $D$ and $L_{d}$ values are in remarkable agreement with experiment, see Table 2.

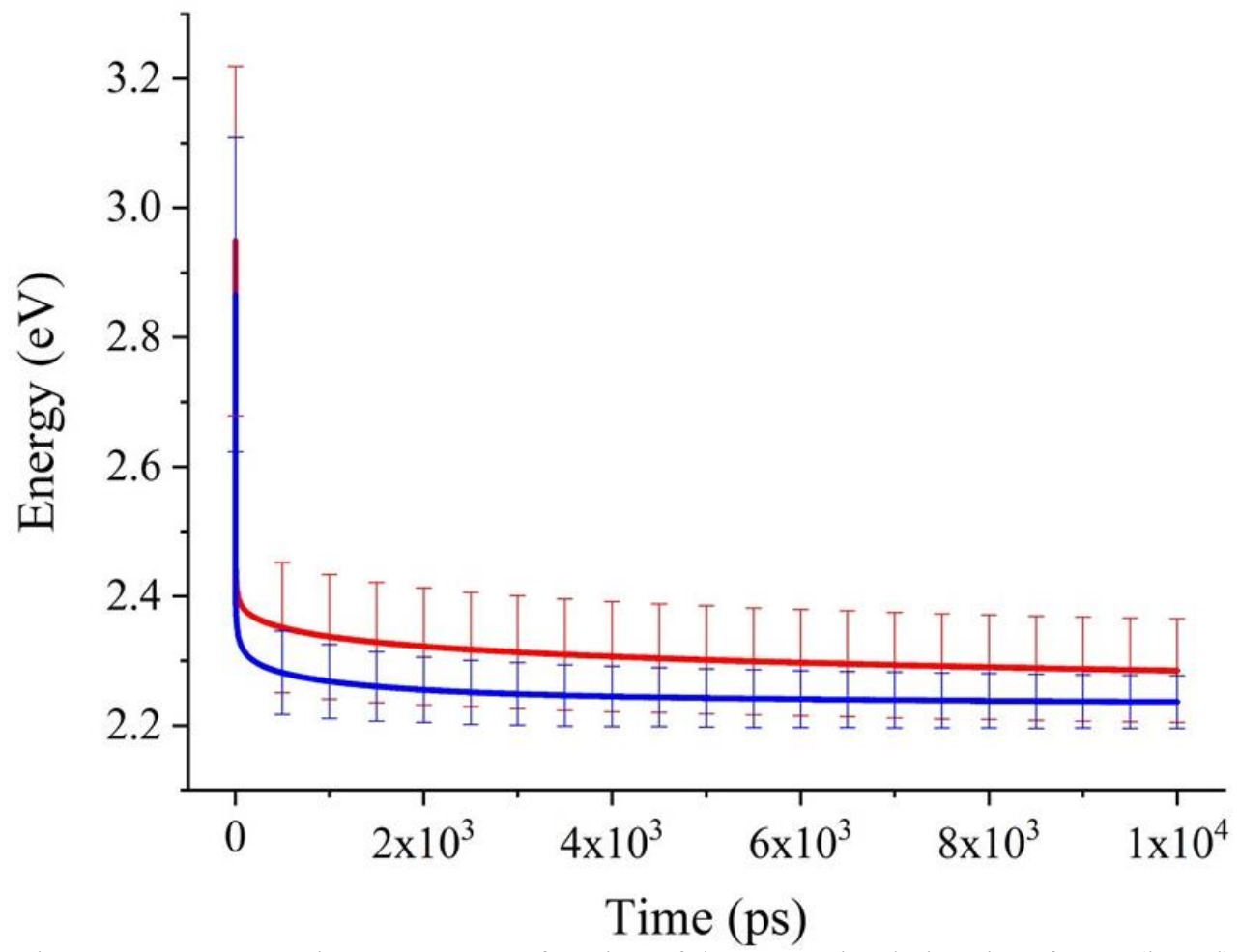

Figure 9: Average exciton energy as a function of the KMC simulation time for $\mathbf{1 a}$ (in red) and $2 \mathbf{a}$ (in blue). Data set standard deviation is represented by vertical bars.

We discussed before the origin for the difference in exciton lifetimes between the two molecules, namely the various amount of charge-transfer admixture into the lowest electronic excitations. In view of the very similar excitonic coupling and rate distributions shown on Figures 6 and 8 respectively, it is, however, not obvious why 1 features an improved diffusion coefficient over 2 . We believe this is routed in the more efficient percolation pathways available for excitons in the shorter molecule, in analogy with the random resistance network for charge transport in organics(39). To test this idea, we assessed the averaged length covered by excitons that hop between molecular sites at a given rate. The corresponding distributions are shown for the two molecules in Figure 11, considering hopping frequencies for the most likely events (in the range of $\left.10^{9}-10^{11} \mathrm{~s}^{-1}\right)$. The distributions are clearly shifted towards longer distances for $\mathbf{1}$ compared to 2, rationalizing the difference in diffusion coefficient. Thus, it appears that there are more long-distance hopping pathways the excitons can funnel through in the smaller compound $\mathbf{1}$, presumably as a result of its reduced anisotropic shape favouring 3D transport.

To close this part, we would like to come back to the non-equilibrium nature of singlet exciton transport in these molecular materials. As previously proposed by Bässler and co-workers(40), a useful set of numerical experiments to probe energetic disorder in organics is to run diffusion simulations using energy-selective 

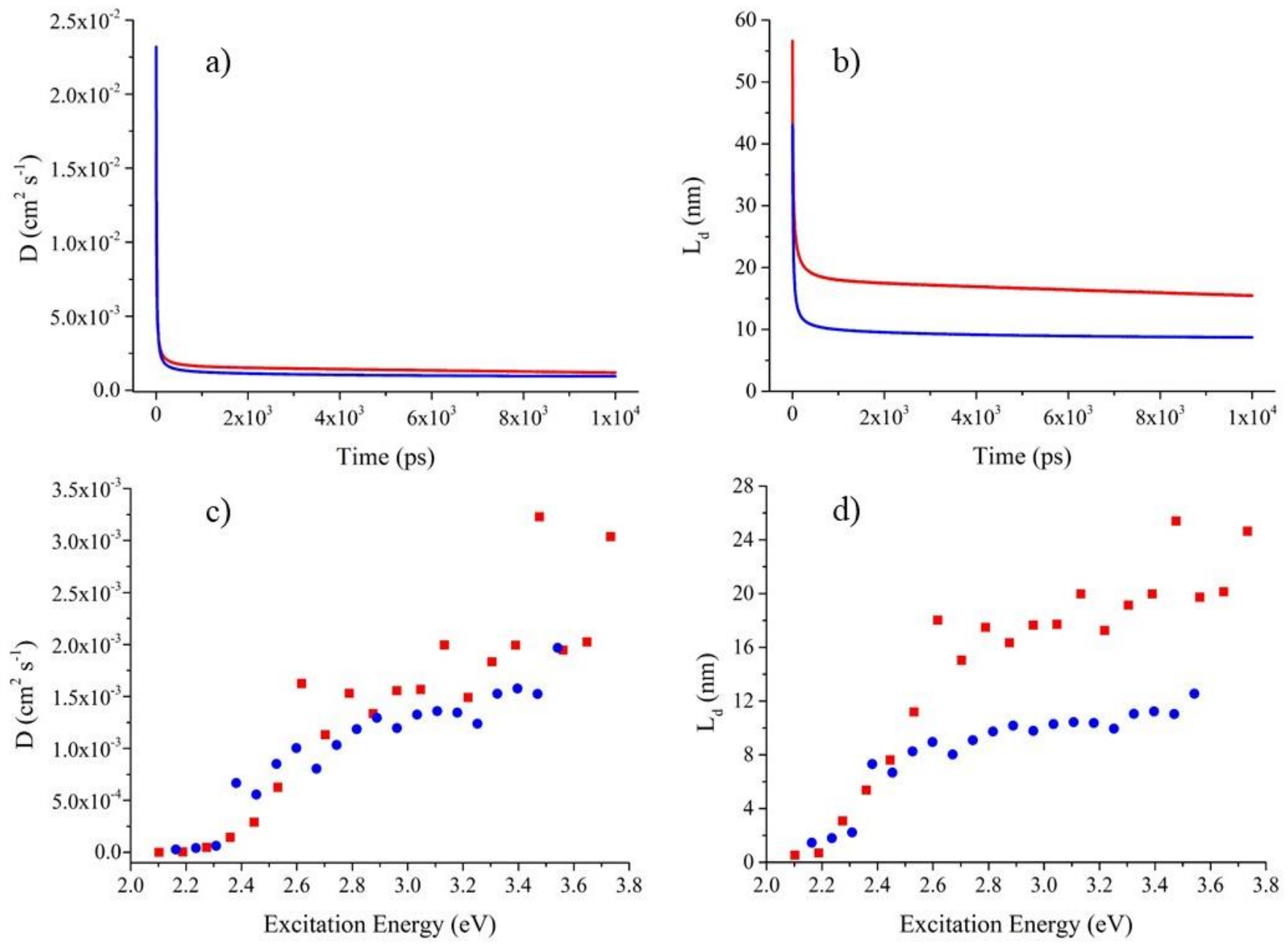

Figure 10: In the upper panel, the calculated a) diffusion coefficient, $D$, and b) exciton diffusion length, $L_{d}$, for $1 \mathbf{a}$ (in red) and $\mathbf{2 a}$ (in blue). In the bottom panel, the same quantities, respectively in c) and in d), were calculated using the 'site-selective' excitations method.

excitations, namely only photo-generating excitons on molecular sites matching a given energy window. Consistent with the disorder model, Figure 10c and 10d shows a strong dependence of the 'effective' diffusion coefficient and length with excitation energy. While high-energy excitons can easily find electronically coupled nearest neighbours with lower energy and thus undergo fast downhill migration, excitons at the bottom of the DOS are instead surrounded by a smaller number of thermally accessible sites and move via slow uphill hopping events.

\section{Conclusions}

We have performed a comprehensive theoretical investigation of the nature and dynamics of electronic excitations in two small $\pi$-conjugated organic molecules that differ only by one thiophene ring in their bridging unit. Earlier experimental reports point to a somewhat unexpected increase in PCE for 1 with respect to $\mathbf{2}$ in vacuum-evaporated OSCs using fullerenes as acceptors $(9,10)$, a result that was explained 

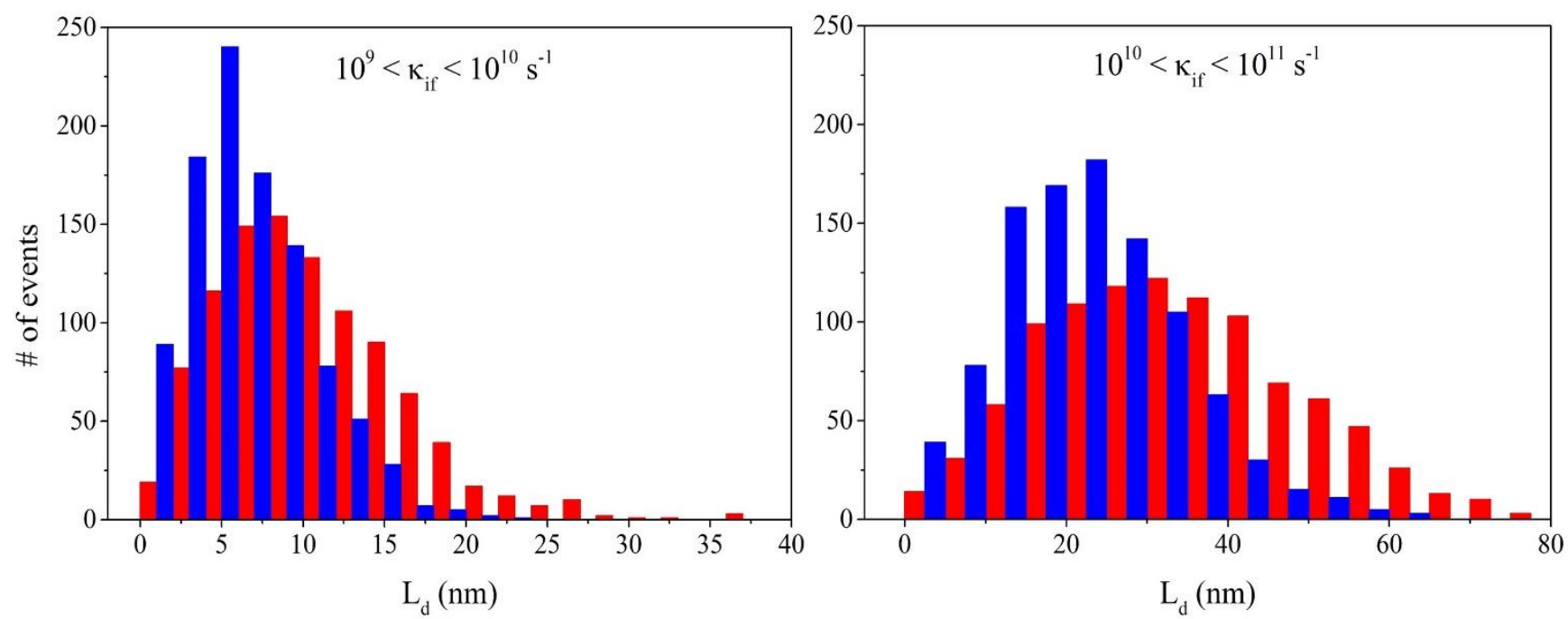

Figure 11: Percolation pathways calculated in two different ranges of exciton hopping rates for 1a (in red) and 2a (in blue).

by the improved singlet exciton diffusion in 1. It was tentatively concluded that the larger diffusion coefficient and diffusion length in the shorter molecule stem from a longer exciton lifetime and a reduced energetic disorder. Our multiscale computation approach, combining MD, TD-DFT and ME calculations to KMC simulations, yields similar overall conclusions, yet for slightly different reasons. The MD/TDDFT/ME results show that the lowest singlet electronic excitations in these molecules have a mixed localized/charge-transfer nature, which largely fluctuates in time and space as a result of conformational motion. On average 1 displays a slightly more pronounced CT admixture, which explains why it absorbs at about the same wavelength as $\mathbf{2}$, despite its shorter conjugated backbone. As a consequence of its increased CT character, 1 features a longer (radiative) lifetime compared to $\mathbf{2}$, in line with the experimental findings and in part responsible for the extended exciton diffusion lengths. The full spectral linewidths of the excitation energy distributions are very large $(>0.2 \mathrm{eV})$ in both molecules because of both conformation and dielectric disorder in the amorphous films. In such a disordered energy landscape, exciton diffusion proceeds largely in a non-equilibrium regime during the finite excited-state lifetime. Close to equilibrium, the diffusion coefficient predicted from KMC simulations is larger for $\mathbf{1}$ compared to $\mathbf{2}$, although the total energetic disorder computed for the two molecules is comparable. The difference in exciton migration in fact reflects the existence of long-range, efficient percolation pathways, likely arising from the slightly less anisotropic shape of $\mathbf{1}$ prompting three-dimensional transport. As a concluding remark, it is important to highlight that the amount of energy disorder actually probed by ps transient experiments as performed in (10) is only a fraction of the total disorder, because in addition to the ultrafast energy drop at very early timescales that requires sub-ps resolution, full energy relaxation has not completed during the exciton lifespan. Rather, fs-resolved photoluminescence measurements using site-selective excitations are required to cover the broad dynamic range in energy distribution present in these inherently disordered soft 
molecular materials. Despite, or perhaps thanks to, their large energetic disorder, molecules such as 1 appear to be very promising candidates for energy photo-conversion. We speculate that, in analogy with electron transport in fullerenes and some of the novel non-fullerene acceptors (REFs), it is the 3D excitonic conductivity of $\mathbf{1}$ that is key to drive long-range exciton motion at dissociation spots.

\section{Conflicts of interest}

There are no conflicts to declare.

\section{Acknowledgements}

The work in Mons was supported by the European Union's Horizon 2020 research and innovation program under Marie Sklodowska Curie Grant agreement No.722651 (SEPOMO). Computational resources were provided by the Consortium des Équipements de Calcul Intensif (CÉCI), funded by the Fonds de la Recherche Scientifiques de Belgique (F.R.S.-FNRS) under Grant No. 2.5020.11, as well as the Tier-1 supercomputer of the Fédération Wallonie-Bruxelles, infrastructure funded by the Walloon Region under Grant Agreement No. 1117545. G.L. acknowledges Dr. V. Lemaur for fruitful discussion. D.B. is a FNRS Research Director.

\section{Notes and references}

1. Bureš F. Fundamental aspects of property tuning in push-pull molecules. RSC Adv. 2014;4(102):58826-51.

2. Che X, Li Y, Qu Y, Forrest SR. High fabrication yield organic tandem photovoltaics combining vacuum- and solution-processed subcells with 15\% efficiency. Nat Energy. 2018 May $23 ; 3(5): 422-7$.

3. Li S, Ye L, Zhao W, Yan H, Yang B, Liu D, et al. A Wide Band Gap Polymer with a Deep Highest Occupied Molecular Orbital Level Enables 14.2\% Efficiency in Polymer Solar Cells. J Am Chem Soc. 2018 Jun 13;140(23):7159-67.

4. O’Regan B, Grätzel M. A low-cost, high-efficiency solar cell based on dye-sensitized colloidal TiO2 films. Nature. 1991;353(6346):737-40. 
5. Allard S, Forster M, Souharce B, Thiem H, Scherf U. Organic Semiconductors for SolutionProcessable Field-Effect Transistors (OFETs). Angew Chemie Int Ed. 2008 May 19;47(22):407098.

6. Ohmori Y. Development of organic light-emitting diodes for electro-optical integrated devices. Laser Photon Rev. 2010 Feb 20;4(2):300-10.

7. Hains AW, Liang Z, Woodhouse MA, Gregg BA. Molecular Semiconductors in Organic Photovoltaic Cells. Chem Rev. 2010 Nov 10;110(11):6689-735.

8. Bakiev AN, Selivanova DG, Lunegov I V, Vasyanin AN, Maiorova OA, Gorbunov AA, et al. Novel thiophene-containing push-pull chromophores that include carbazole and triphenylamine moieties: study of optical and electrochemical properties. Chem Heterocycl Compd. 2016;52(6):379-87.

9. Kozlov O V, Liu X, Luponosov YN, Solodukhin AN, Toropynina VY, Min J, et al. Triphenylamine-Based Push-Pull Molecule for Photovoltaic Applications: From Synthesis to Ultrafast Device Photophysics. J Phys Chem C. 2017 Mar 30;121(12):6424-35.

10. Kozlov O V, Luponosov YN, Solodukhin AN, Flament B, Douhéret O, Viville P, et al. Simple donor-acceptor molecule with long exciton diffusion length for organic photovoltaics. Org Electron. 2018;53:185-90.

11. Athanasopoulos S, Emelianova E V, Walker AB, Beljonne D. Exciton diffusion in energetically disordered organic materials. Phys Rev B. 2009 Nov 19;80(19):195209.

12. Athanasopoulos S, Hennebicq E, Beljonne D, Walker AB. Trap Limited Exciton Transport in Conjugated Polymers. J Phys Chem C. 2008 Jul 1;112(30):11532-8.

13. Mayo SL, Olafson BD, Goddard WA. DREIDING: a generic force field for molecular simulations. J Phys Chem. 1990 Dec 1;94(26):8897-909.

14. Courtesy of Enikolopov Institute of Synthetic Polymeric Materials of the Russian Academy of Sciences.

15. M. J. Frisch, G. W. Trucks, H. B. Schlegel, G. E. Scuseria, M. A. Robb, J. R. Cheeseman, G. Scalmani, V. Barone, B. Mennucci, G. A. Petersson, H. Nakatsuji, M. Caricato, X. Li, H. P. Hratchian, A. F. Izmaylov, J. Bloino, G. Zheng, J. L. Sonnenberg, M. Had 2009. Gaussian 09, Revision D.01. 
16. Stephens PJ, Devlin FJ, Chabalowski CF, Frisch MJ. Ab Initio Calculation of Vibrational Absorption and Circular Dichroism Spectra Using Density Functional Force Fields. J Phys Chem. 1994 Nov 1;98(45):11623-7.

17. Adamo C, Barone V. Toward reliable density functional methods without adjustable parameters: The PBE0 model. J Chem Phys. 1999 Mar 23;110(13):6158-70.

18. Kronik L, Stein T, Refaely-Abramson S, Baer R. Excitation Gaps of Finite-Sized Systems from Optimally Tuned Range-Separated Hybrid Functionals. J Chem Theory Comput. 2012 May $8 ; 8(5): 1515-31$.

19. Körzdörfer T, Brédas J-L. Organic Electronic Materials: Recent Advances in the DFT Description of the Ground and Excited States Using Tuned Range-Separated Hybrid Functionals. Acc Chem Res. 2014 Nov 18;47(11):3284-91.

20. Yanai T, Tew DP, Handy NC. A new hybrid exchange-correlation functional using the Coulombattenuating method (CAM-B3LYP). Chem Phys Lett. 2004;393(1):51-7.

21. Chai J-D, Head-Gordon M. Long-range corrected hybrid density functionals with damped atomatom dispersion corrections. Phys Chem Chem Phys. 2008;10(44):6615-20.

22. Chai J-D, Head-Gordon M. Systematic optimization of long-range corrected hybrid density functionals. J Chem Phys. 2008 Feb 27;128(8):84106.

23. Do K, Ravva MK, Wang T, Brédas J-L. Computational Methodologies for Developing StructureMorphology-Performance Relationships in Organic Solar Cells: A Protocol Review. Chem Mater. 2017 Jan 10;29(1):346-54.

24. Sun H, Zhong C, Brédas J-L. Reliable Prediction with Tuned Range-Separated Functionals of the Singlet-Triplet Gap in Organic Emitters for Thermally Activated Delayed Fluorescence. J Chem Theory Comput. 2015 Aug 11;11(8):3851-8.

25. D’Avino G, Muccioli L, Zannoni C, Beljonne D, Soos ZG. Electronic Polarization in Organic Crystals: A Comparative Study of Induced Dipoles and Intramolecular Charge Redistribution Schemes. J Chem Theory Comput. 2014 Nov 11;10(11):4959-71.

26. D'Avino G, Muccioli L, Castet F, Poelking C, Andrienko D, Soos ZG, et al. Electrostatic phenomena in organic semiconductors: Fundamentals and implications for photovoltaics. J Phys Condens Matter. 2016;28(43). 
27. Olivier Y, Yurash B, Muccioli L, D’Avino G, Mikhnenko O, Sancho-García JC, et al. Nature of the singlet and triplet excitations mediating thermally activated delayed fluorescence. Phys Rev Mater. 2017 Dec 27;1(7):75602.

28. Besler BH, Merz Jr. KM, Kollman PA. Atomic charges derived from semiempirical methods. J Comput Chem. 1990 May 1;11(4):431-9.

29. Jortner J. Temperature dependent activation energy for electron transfer between biological molecules. J Chem Phys. 1976 Jun 15;64(12):4860-7.

30. Hsu C-P, You Z-Q, Chen H-C. Characterization of the Short-Range Couplings in Excitation Energy Transfer. J Phys Chem C. 2008 Jan 1;112(4):1204-12.

31. Head-Gordon M, Grana AM, Maurice D, White CA. Analysis of Electronic Transitions as the Difference of Electron Attachment and Detachment Densities. J Phys Chem. 1995 Sep 1;99(39):14261-70.

32. Gillespie DT. A general method for numerically simulating the stochastic time evolution of coupled chemical reactions. J Comput Phys. 1976;22(4):403-34.

33. Olivier Y, Sancho-Garcia J-C, Muccioli L, D’Avino G, Beljonne D. Computational Design of Thermally Activated Delayed Fluorescence Materials: The Challenges Ahead. J Phys Chem Lett. 2018 Oct 18;9(20):6149-63.

34. Evans EW, Olivier Y, Puttisong Y, Myers WK, Hele TJH, Menke SM, et al. Vibrationally Assisted Intersystem Crossing in Benchmark Thermally Activated Delayed Fluorescence Molecules. J Phys Chem Lett. 2018 Jul 19;9(14):4053-8.

35. Kozlov O V, Luponosov YN, Ponomarenko SA, Kausch-Busies N, Paraschuk DY, Olivier Y, et al. Ultrafast Charge Generation Pathways in Photovoltaic Blends Based on Novel Star-Shaped Conjugated Molecules. Adv Energy Mater. 2015 Apr 1;5(7):1401657.

36. Castet F, D’Avino G, Muccioli L, Cornil J, Beljonne D. Charge separation energetics at organic heterojunctions: on the role of structural and electrostatic disorder. Phys Chem Chem Phys. 2014;16(38):20279-90.

37. D’Avino G, Olivier Y, Muccioli L, Beljonne D. Do charges delocalize over multiple molecules in fullerene derivatives? J Mater Chem C. 2016;4(17):3747-56.

38. Vallée RAL, Van Der Auweraer M, De Schryver FC, Beljonne D, Orrit M. A Microscopic Model 
for the Fluctuations of Local Field and Spontaneous Emission of Single Molecules in Disordered Media. ChemPhysChem. 2005 Jan 14;6(1):81-91.

39. Sirringhaus H. 25th Anniversary Article: Organic Field-Effect Transistors: The Path Beyond Amorphous Silicon. Adv Mater. 2014 Mar 1;26(9):1319-35.

40. Bässler H, Schweitzer B. Site-Selective Fluorescence Spectroscopy of Conjugated Polymers and Oligomers. Acc Chem Res. 1999 Feb 1;32(2):173-82. 